\title{
Original
}

\section{HGF and IGF-1 is Present during the Developmental Process of Murine Masseter Muscle}

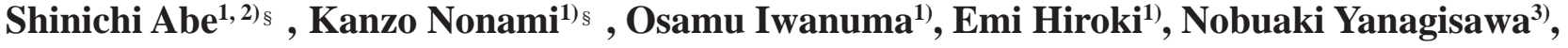 \\ Koji Sakiyama4) and Yoshinobu Ide ${ }^{1)}$
}

\begin{abstract}
1) Department of Anatomy, Tokyo Dental College 1-2-2 Masago, Mihama-ku, Chiba-City, Chiba 261-8502, Japan
2) Oral Health Science Center HRC7, Tokyo Dental College 1-2-2 Masago, Mihama-ku, Chiba-City, Chiba 261-8502, Japan

3) Department of Oral Anatomy, Showa University School of Dentistry, 1-5-8 Hatanodai, Shinagawa-ku, Tokyo 142-8555, Japan

4) Division of Anatomy, Department of Human Development and Fostering, Meikai University School of Dentistry, 1-1 Keyakidai, Sakado-City, Saitama 350-0283, Japan
\end{abstract}

(Accepted for publication, January 15, 2009)

\begin{abstract}
Until now the developmental process of masticatory muscle (derived from the branchial arches) is not well documented. Thus, in order to clarify the developmental process of murine masseter muscle, Hepatocyte Growth Factor (HGF), Insulin-like Growth Factor-1 (IGF-1) and Myosin Heavy Chain (MyHC) were evaluated by histological examination, western blotting and RT-PCR analyses on embryonic days 14 (E14), 16 (E16) and 18 (E18). E14 showed that the mandibular bone development is initiated by the appearance of the ossification centre in the peripheral zone of Meckel cartilage. Whereas, E16 demonstrated presence of developing masseter muscle at the lower part of the mandible composed of immature bone. On the contrary, E18 showed larger masseter muscle and more mature mandibular than E16. Interestingly, both IGF and HGF proteins and genes increased at initial stage of masseter muscle development, and decreased with the time. A significant change in composition of MyHC was detected in E18 compared with E14 and E16. This result indicate that murine masseter muscle develops in accordance with the surrounding tissue, where IGF-1 and HGF could be an important modulator of stem cell-derived myoblast proliferation and myogenic differentiation, and MyHC-2a and 2d are strongly expressed by mature muscle fibres during the developmental process of the masseter muscle.
\end{abstract}

Key words: IGF-1, HGF, MyHC, Masseter muscle, Myogenesis

\section{Introduction}

Skeletal muscle is a type of striated muscle, which are used to create movement by applying force to bones and joints via contraction. The relationship between the combination and the function of the muscle fibers has been published ${ }^{1,2)}$. Skeletal muscles contain two main types of fibers: Slow-twitch or type I and fast-twitch or type II according to their different speeds of contraction ${ }^{3)}$.

There are two types of isoforms of muscle contractile protein known as myosin heavy chain (MyHC): the fast-type isoforms MyHC-2b, MyHC-2d, and MyHC-2a; and the slow-type isoform, MyHC-14). The functional roles of these four isoforms have been clarified; therefore, the functions of muscles can be elucidated by determining the composition of these four isoforms ${ }^{1,5-8)}$.

\footnotetext{
$\S$ These authors contributed equally to this work. Correspondence to: Shinichi Abe DDS.PhD,Department of Anatomy, Tokyo Dental College 1-2-2 Masago, Mihama-ku, Chiba-City, Chiba 2618502, Japan. Tel: +81-43-270-3571, Fax: +81-43-277-4010; E-mail: abesh@tdc.ac.jp
}

Many reports have been published on the developmental processes of muscles in the trunk and the legs ${ }^{9,10}$. Lyons et al. (1990) and Sartorius et al. (1998) studied the gene expressions of MyHC isoforms at the pre and post-natal stages in murine somites and in murine hindlimbs, respectively ${ }^{11,12)}$. On the other hand, there are only a few reports on masticatory muscles that are derived from the branchial arches ${ }^{13)}$. It has been reported that the masseter muscle, which is one of the masticatory muscles, expresses different compositions of MyHC isoforms compared with the tibialis anterior during the transitional phase from suckling to mastication ${ }^{14}$. Contractile protein isoforms expressed only at the pre and post-natal stages were detected in masseter muscle ${ }^{15)}$. However, the developmental process of masseter muscle is not well documented yet.

On the other hand, it is well known that various growth factor thought to play a role in different stages of muscle regeneration. Thus, hepatocyte growth factor (HGF) affect satellite cell activation $^{16)}$ and proliferation ${ }^{17,18)}$; Insulin Growth Factor-1 and 2 
Shinichi Abe et al.: IGF-1, HGF and MyHC are Expressed in Masseter Muscle Development.

(IGF-1 and IGF-2) stimulates both proliferation and differentiation ${ }^{18)}$. Until now, the expression of HGF and IGF during masseter muscle development has not been reported.

For that reason in the present study, expression HGF, IGF-1 and MyHC isoforms of the masseter muscle fibers of embryonic stage were analyzed.

\section{Materials and Methods}

\section{Specimens}

A total of 25 samples of ICR mice (Sankyo Laboratory, Tokyo, Japan) were used in this study. The mice were used on embryonic days 14, 16 and 18 of pregnancy. The mice were anesthetized with pentobarbital, and sacrificed according to the Guidelines for Animal Experiments of Tokyo Dental College. Foetuses taken from the uterus were decapitated, and 5 specimens were examined by hematoxylin-eosin (H-E) staining, 10 samples for western blotting and 10 samples of RT-PCR analysis.

\section{Morphological examination}

The masseter muscle together with the mandible were removed and fixed with $4 \%$ paraformaldehide. Then, the specimens were dehydrated, embedded in paraffin using Tissue-Tech VIP M1500, and cut at $4 \mu \mathrm{m}$ in thickness in serial sections with a microtome (IVS-410). After deparaffin treatment, the samples were rinsed with water and stained with a routine hematoxylin-eosin (H-E).

Western blotting analysis of Insulin-like Growth Factor-1 (IGF1) and Hepatocyte Growth Factor (HGF)

Electrophoretic separation and analysis of the protein bands by western blotting was performed. Briefly, the masseter muscle at each observation period was removed. The specimens were weighed, frozen in liquid nitrogen, and stored at $-80^{\circ} \mathrm{C}$. Frozen muscles were minced with scissors in nine volumes of ice-cold homogenization buffer. The minced muscle samples were subsequently sonicated in a Branson Sonifier 250D (Branson Ultrasonic Corporation, Danbury, CT, USA). The products were used for the preparation of washed myofibers, which were then boiled in sample buffer for $2 \mathrm{~min}$ at a final protein concentration of $0.125 \mathrm{mg} / \mathrm{ml}$. Total proteins were determined by the Bradford technique using the Bio-Rad Protein Assay (Nippon Bio-Rad Laboratory) and a Gene Quantpro spectrophotometer (Amersham Pharmacia Biotech Inc., Piscataway, NJ, USA). Western blotting analyses were performed for the detection of IGF-1 and HGF. Equal amount of total protein for each group $(0.5 \mu \mathrm{g})$ was separated on $12.5 \%$ SDS-polyacrylamide gel and was transferred to Immobilon P membrane (Millipore, Bedford, MA, USA). Membranes were blocked with 5\% skimmed milk and incubated with each of primary antibodies of anti-IGF-1 (1:1000, LVC MS1508-PO), anti-HGF (1:500, ADV 905-165), and detected with horseradish peroxidase-conjugated secondary anti-rabbit/mouse IgG antibody using the VECTASTAIN ABC Rabbit IgG Kit.
Table 1 The primers used for the RT-PCR analysis

\begin{tabular}{ll}
\hline IGF-1 Forward 5'- TCGTCTTCACACCTCTTCTA -3' & , \\
& Reverse 5'- AAGCAACACTCATCCACAAT -3,
\end{tabular}

HGF Forward 5'-CACCCCTTGGGAGTATTGTG -3' Reverse 5'- GGGACATCAGTCTCATTCACAG -3'

MyHC-1 Forward 5'- GAGTCCCAGGTCAACAAGC -3' Reverse 5'- AACCCAGAGAGGCAAGTGAC -3'

MyHC-2a Forward 5'- CGATGATCTTGCCAGTAATG -3’ Reverse 5'- ATAACTGAGATACCAGCG -3'

MyHC-2d Forward 5'- GACAAACTGCAATCAAAGG -3' Reverse 5'- TTGGTCACTTTCCTGCACTT -3'

MyHC-2b Forward 5' - ACAGACTAAAGTGAAAGCC -3' Reverse 5'- CTCTCAACAGAAAGATGGAT -3'

GAPDH Forward 5'-TGAACGGGAAGCTCACTGG -3' Reverse 5'-TCCACCACCCTGTTGCTGTA -3'

GAPDH Ct $=19, \mathrm{IGF}-1 \mathrm{Ct}=24, \mathrm{HGF} \mathrm{Ct}=24, \mathrm{MyHC}-1 \mathrm{Ct}=21$, MyHC-2a Ct $=21$, MyHC-2d Ct =22, MyHC-2b Ct $=23$.

\section{Reverse transcription polymerase chain reaction analysis}

From mice in each age group, the muscle was removed and snap-frozen in liquid nitrogen. mRNA was extracted using a QuickPrep micro mRNA Purification Kit (Amersham Pharmacia Biotech UK Ltd., Buckinghamshire, UK), and cDNA was prepared using a Ready-To-Go kit (Amersham Pharmacia Biotech UK Ltd.). After determination of the optimal PCR conditions for all primers, experiments were performed using a LightCycler ${ }^{\mathrm{TM}}$ (Roche Diagnostics, Mannheim, Germany), which allows RNA quantification. The two primer sets designed from the sequences of IGF-1 HGF, MyHC-1, MyHC-2a, MyHC-2d and MyHC-2b genes were used. Experiments were performed according to the standard protocol for the LightCycler ${ }^{\mathrm{TM}}$. A ready-to-use LC FastStart DNA Master SYBR Green I (Roche) was used as a hotstart PCR reaction mixture for the LightCycler ${ }^{\mathrm{TM}}$. A series of cDNA dilutions (4.0 ng/ $\mu \mathrm{l}$ ) including $1 / 10^{5}, 1 / 10^{6}, 1 / 10^{7}, 1 / 10^{8}$, and $1 /$ $10^{9}$ were prepared. PCR reaction for the diluted standards contained $10.2 \mu \mathrm{l}$ of sterile water, $5 \mu \mathrm{l}$ of diluted control cDNA product, $1.6 \mu \mathrm{l}$ of $25 \mathrm{mM} \mathrm{MgCl}_{2}$, and $2 \mu \mathrm{l}$ of LC FastStart DNA Master SYBR Green I containing SYBR Green I (1/60,000 dilution). In addition, $0.6 \mu \mathrm{l}$ of each of forward and reverse primers designed by the software (Biogene Ltd.) were added to reach a final reaction volume of $20 \mu \mathrm{l}$ for each tube. The primers based on sequences of IGF-1 HGF, MyHC-1, MyHC-2a, MyHC-2d and MyHC-2b genes were designed from specific segments of the entire DNA sequence (Table 1).

Each PCR mixture (final reaction volume, $20 \mu \mathrm{l}$ ) contained 14.2 ìl of sterile water, $1.6 \mu \mathrm{l}$ of $25 \mathrm{mM} \mathrm{MgCl}_{2}$, SYBR Green I (1/ 60,000 dilution), $2 \mu \mathrm{l}$ of (DNA (5 pg/ $\mu \mathrm{l}$ )- containing LC FastStart DNA Master SYBR Green I, $0.6 \mu \mathrm{l}$ of forward primer (10 pmol/ 

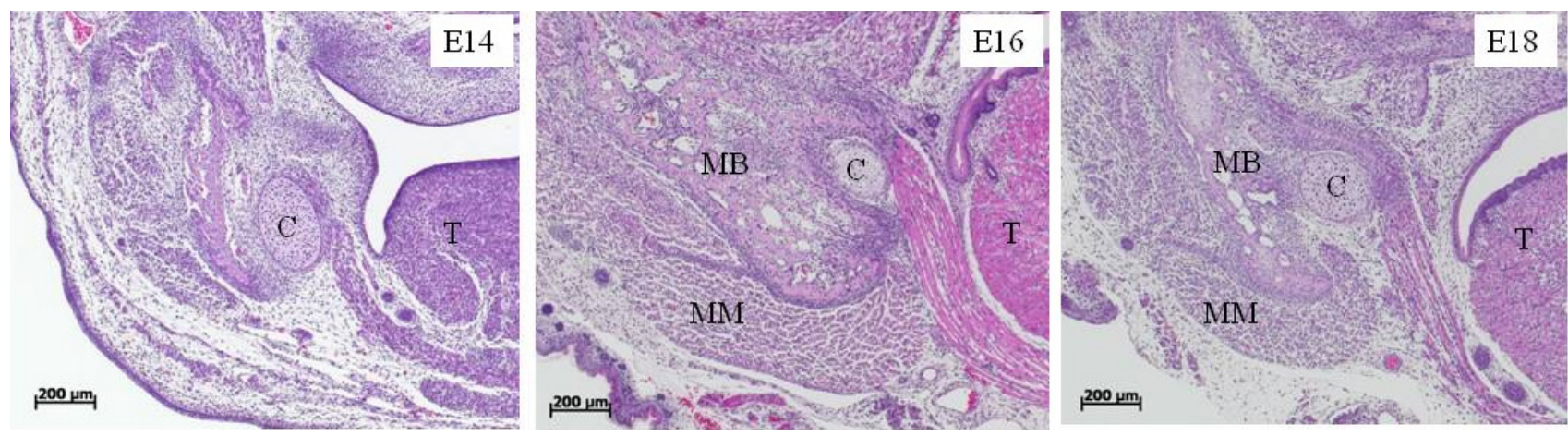

Figure 1. Histological examination of surgical section of the mandible on embryonic days 14 (E14), 16 (E16) and 18 (E18). In E14 there was no formation neither of masseter muscle nor mandible. On the contrary, presence of developing masseter muscle at the lower part of the mandible was detected in E16 and E18. H-E staning, T: tongue, C: cartilage, MB: mandible, MM: masseter muscle.

$\mu \mathrm{l}), 0.6 \mu \mathrm{l}$ of reverse primer $(10 \mathrm{pmol} / \mu \mathrm{l})$ and $1 \mu \mathrm{l}$ of target DNA. The PCR mixtures (20 $\mu \mathrm{l}$ each) prepared for IGF-1 HGF, MyHC1, MyHC-2a, MyHC-2d and MyHC-2b were added to the glass portion of capillary. Initial denaturation at $95^{\circ} \mathrm{C}$ for $10 \mathrm{~min}$ was followed by 45 cycles of a denaturation step at $95^{\circ} \mathrm{C}$ for $10 \mathrm{~s}$, an annealing step at $60^{\circ} \mathrm{C}$ for 10 s, and an extension step at $72^{\circ} \mathrm{C}$ for $80 \mathrm{~s}$. Gene amplification was performed according to a melting program of $70^{\circ} \mathrm{C}$ for $15 \mathrm{~s}$, and fluorescence was continuously monitored at a rate of $0.1^{\circ} \mathrm{C} / \mathrm{s}$.

Amount of each gene final expression was obtained by dividing each of the gene expression level with housekeeping gene GAPDH level. The cycle threshold values (Ct values) of the real time data for each gene were found to be: GAPDH Ct $=19$, IGF-1 Ct $=24$, HGF Ct =24, MyHC-1 Ct =21, MyHC-2a Ct $=21$, MyHC-2d Ct $=22$ and MyHC-2b Ct $=23$. The primers for GAPDH had the following sequences shown in the table 1.

\section{Statistical analysis}

Student's t-test was used for statistical analysis in this study, and the $p$ value of $<0.05$ was designated as significant.

\section{Results}

\section{Histological examination}

In order to evaluate the histopathological findings, the samples

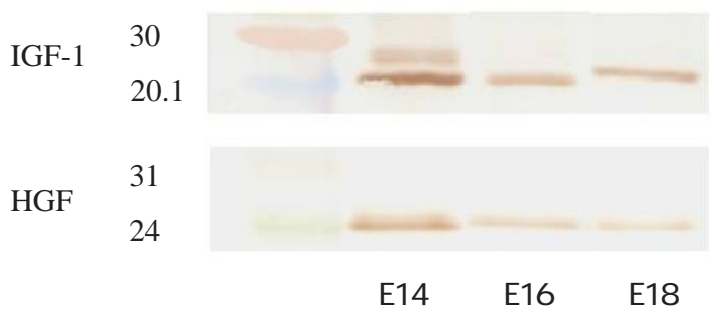

Figure 2. Western blot analysis of IGF-1 and HGF in masseter muscle on embryonic days 14 (E14), 16 (E16) and 18 (E18). Note strong IGF-1 and HGF protein expression in E14, which were gradually decreased in E16 and E18.

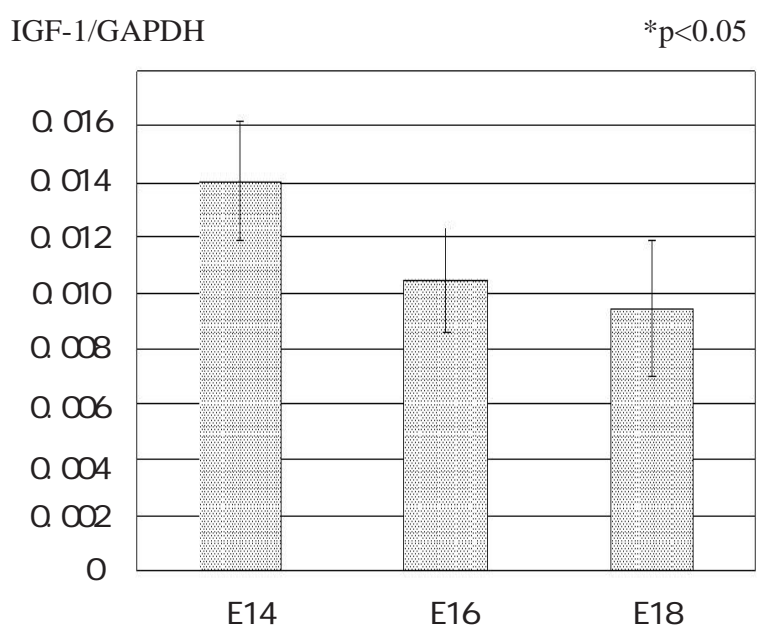

Figure 3. Expression of IGF-1 mRNA in masseter muscle on embryonic days 14 (E14), 16 (E16) and 18 (E18). A maximum IGF-1 mRNA expression was detected in E14, with a marked decrease in E16 and E18. Interestingly, E14 demonstrated a significant increase in IGF-1 expression compared to E16 and E18 ( $n=10, \mathrm{p}<0.05)$.

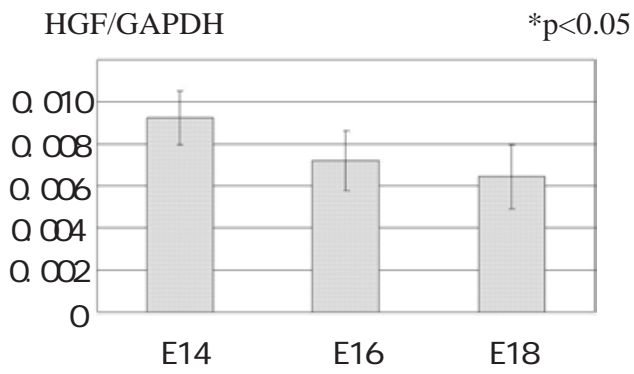

Figure 4. Expression of HGF mRNA in masseter muscle on embryonic days 14 (E14), 16 (E16) and 18 (E18). A maximum HGF mRNA expression was detected in E14, with a marked decrease in E16 and E18. Interestingly, E14 demonstrated a significant increase in HGF expression compared to E16 and E18 $(n=10, p<0.05)$.

were stained with H-E (Fig.1). At early stage, E14 did not show any evidence of neither masseter muscle nor mandible. The 
Shinichi Abe et al.: IGF-1, HGF and MyHC are Expressed in Masseter Muscle Development.
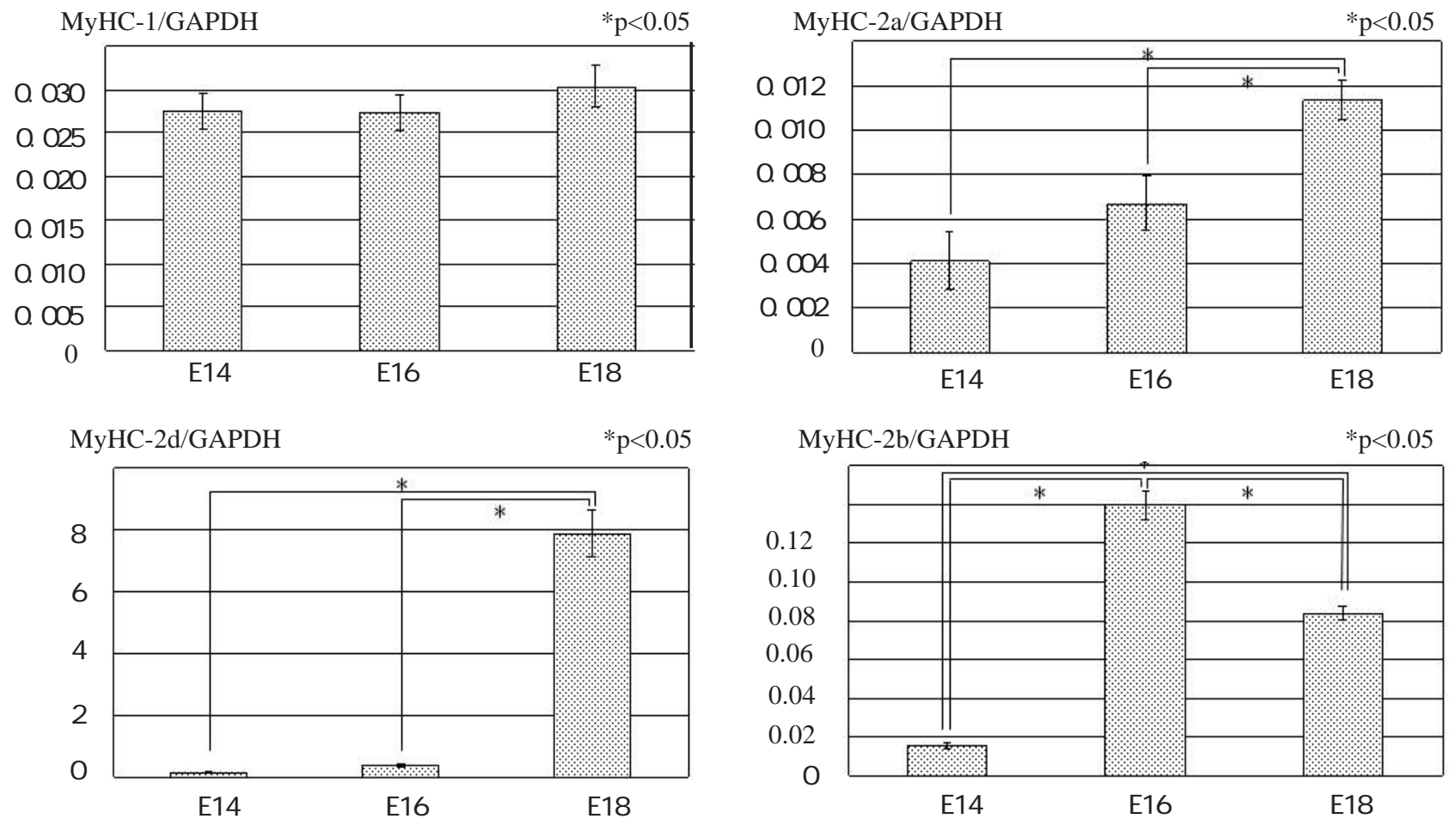

Figure 5. Expression of four MyHC isoforms in masseter muscle at various embryonic stages. MyHC-1 did not show any significant difference in E14, E16 and E18. However, MyHC-2a and MyHC-2d demonstrated a gradually increase from E14 to E18. A maximum expression was detected in E18, with significant increase in MyHC-2a and MyHC-2d expression compared to E14 and E16 ( $n=10, p<0.05)$. While, MyHC-2b showed a maximum expression in E16, with statistically difference compared to E14 and E18 ( $n=10, p<0.05)$.

mandibular bone development was initiated by the appearance of the ossification centre in the peripheral zone of Meckel cartilage during this initial stage. Whereas, E16 demonstrated presence of developing masseter muscle at the lower part of the mandible, which was composed of immature bone. On the contrary, mandibular bone observed in E18 is actively calcified and more mature than E16.

\section{Expression of IGF-1 and HGF proteins}

IGF-1 and HGF proteins were identified in the masseter muscle at various embryonic stages (Fig.2). Results indicated that IGF-1 and HGF were detected in all period of time. The bands immunoreactive to these proteins were strongly expressed at 14 days. However, both proteins expression were weakly expressed at 16 and 18 days.

\section{Quantitative analysis of IGF-1, HGF and MyHC genes}

To determine whether IGF-1, HGF and MyHC mRNA are expressed in the masseter muscle at 14, 16 and 18 days during embryonic stage, total RNA was isolated from the masseter muscle and evaluated by RT-PCR analysis. Figure 3 and 4 summarize IGF-1 and HGF mRNA quantification of masseter muscle on embryonic days 14, 16 and 18. Both genes gradually decreased from 14 to 18 days. Figure 5 summarize expression of four MyHC isoforms in masseter muscle at various embryonic stages. MyHC1 did not show any significant difference in E14, E16 and E18 groups. However, MyHC-2a and MyHC-2d demonstrated a gradually increase from E14 to E18. A maximum expression was detected in E18, with a significant increase in MyHC-2a and MyHC-2d expression compared to E14 and E16 ( $n=10, p<0.05)$. While, MyHC-2b showed a maximum expression in E16, with statistically difference compared to E14 and E18 ( $n=10, p<0.05)$.

\section{Discussion}

Until now, the developmental process of masticatory muscle (derived from the branchial arches) is not well documented. Thus, in order to clarify the developmental process of murine masseter muscle, HGF, IGF-1 and MyGF were examined by western blotting and RT-PCR analyses.

It is well known that skeletal muscle cell differentiation is a process whereby mesodermal stem cell-derived myoblasts cease proliferation and acquire the biochemical and morphologic phenotype of myocytes and fused myotubes ${ }^{19)}$.The decision of myoblasts to proliferate or differentiate is strongly linked to the cellular environment, influenced by cell-extracellular matrix interactions ${ }^{20)}$, direct cell-cell interactions, ${ }^{21)}$ and by soluble growth factors $^{22)}$. Although growth factors, in general, inhibit myogenic differentiation ${ }^{22)}$, IGFs are distinctive in that they are the only 
known mitogens that, when free of other serum components, stimulate both the proliferation and differentiation of skeletal muscle cells ${ }^{18,22)}$. HGF is expressed by activating satellite cells not only in regenerating muscle ${ }^{16,23)}$ but also in normal adult skeletal muscle ${ }^{16)}$. At initial stages after injury, HGF serves to activate quiescent satellite cells ${ }^{17}$. By blocking the differentiation of these activated satellite cells until there are sufficient cell numbers, HGF could serve to increase the pool of proliferating myoblasts to some optimal cell density, whereupon fusion could commence $^{17)}$. Such a model would predict that the levels of HGF should decrease within regenerating muscles. A similar result showed the current study during muscle development, both IGF1 and HGF proteins and genes increase at initial stage, and decrease with the time (Fig.2, 3, and 4). The present experiment strongly suggests that IGF-1 and HGF could be considered as an important modulator of stem cell-derived myoblast proliferation and myogenic differentiation.

Four isoforms of myosin, which belongs to contractile proteins, have been reported: MyHC-2b, MyHC-2d, MyHC-2a and MyHC1. MyHC-2b, MyHC-2d and MyHC-2a are fast-type isoforms, and MyHC-1 is a slow-type isoform ${ }^{12,24)}$.

The adult mouse masseter muscle consists only of fast muscle fibers ${ }^{25,26)}$. Many studies reported time-dependent changes in muscle fiber properties in the fetal masseter muscle ${ }^{5)}$ and the postnatal tongue ${ }^{7}$. Gojo et al. (2002) attempted to elucidate detailed masseter muscle functional changes by examining the relative MyHC isoform composition (MyHC-2a and MyHC-2b), and found that the muscle characteristics changed markedly after weaning, and that these weaning-associated characteristic changes were not observed in extremity muscles ${ }^{14)}$. They speculated that, coincident with the weaning period, MyHC- 2a protein, which was considered to contract quickly and strongly, appeared abundantly, and that the transition to mastication promoted the development of the masseter muscle function. Furthermore, Shida et al. (2005) showed that the superficial and deep layers of the masseter muscle, with fibers running in different directions, differed with regard to the muscle fiber characteristics, suggesting that the superficial layer of the master muscle is more closely involved in masticatory function ${ }^{27}$. On the other hand, Usami et al. (2003) reported that a significant change in composition of MyHC was identified between embryonic days 14 and 16, a stage when murine facial development is conspicuous suggesting that the development of murine masseter muscle is closely associated with facial development ${ }^{5)}$. A similar result was observed in this study with a significant change in composition of MyHC detected in E18 compared with E14 and E16 groups (Fig.5). The histological analysis showed that the mandibular bone development is initiated by the appearance of the ossification centre in the peripheral zone of Meckel cartilage in E14. Whereas, E16 demonstrated presence of developing masseter muscle at the lower part of the mandible, which is composed of immature bone.
On the contrary, mandibular bone observed in E18 is actively calcified and more mature than E16.

\section{Conclusion}

In summary, these results indicate that murine masseter muscle develops in accordance with the surrounding tissue, where, IGF and HGF could be an important modulator of stem cell-derived myoblast proliferation and myogenic differentiation, and MyHC$2 \mathrm{a}$ and $2 \mathrm{~d}$ are strongly expressed by mature muscle fibres during the developmental process of the masseter muscle. Further complementary research will be useful for future clinical application of muscle regeneration and transplantation.

\section{Acknowledgments}

This study was supported by grants-in-aid for scientific research (19592131: Shinichi Abe) from the Ministry of Education, Culture, Sports, Science and Technology, Japan, by the Foundation of Japan Medical Association, by Oral Health Science Center Grant HRC7 (Shinichi Abe) from Tokyo Dental College, and by a "High-Tech Research Center” Project for Private Universities: matching fund subsidy from MEXT (Ministry of Education, Culture, Sports, Science and Technology) of Japan, 2006-2011.

\section{Refernces}

1. Abe S, Maejima M, Watanabe $H$, Shibahara T, Agematsu $H$, Doi T, Sakiyama K, Usami A, Gojyo K, Hashimoto M, Yoshinari $\mathrm{M}$ and Ide Y. Muscle-fiber characteristics in the adult mouse-tongue muscles. Anat Sci Int 77: 145-148, 2002

2. Buonanno Aand Rosenthal N. Molecular control of muscle diversity and plasticity. Dev Genet 19: 95-107, 1996

3. Swynghedauw B. Developmental and functional adaptation of contractile proteins in cardiac and skeletal muscles. Physiol Rev 66: 710-771, 1986

4. Pette D and Staron RS. Cellular and molecular diversities of mammalian skeletal muscle fibers. Rev Physiol Biochem Pharmacol 116: 1-76, 1990

5. Usami A, Abe S and Ide Y. Myosin heavy chain isoforms of the murine masseter muscle during pre- and post-natal development. Anat Histol Embryol 32: 244-248, 2003

6. Doi T, Abe $S$ and Ide Y. Masticatory function and properties of masseter muscle fibers in microphthalmia (mi/mi) mice during postnatal development. Ann Anat 185: 435-440, 2003

7. Maejima M, Abe S, Sakiyama K, Agematsu H, Hashimoto $\mathrm{M}$, Tamatsu Y and Ide Y. Changes in the properties of mouse tongue muscle fibres before and after weaning. Arch Oral Biol 50: 988-993, 2005

8. Sakiyama K, Abe S, Tamatsu Y and Ide Y. Effects of stretching stress on the muscle contraction proteins of skeletal muscle myoblasts. Biomed Res 26: 61-68, 2005

9. Rubinstein NA and Kelly AM. Development of muscle fiber specialization in the rat hindlimb. J Cell Biol 90: 128-144, 
Shinichi Abe et al.: IGF-1, HGF and MyHC are Expressed in Masseter Muscle Development.

1981

10. Whalen RG, Sell SM, Butler-Browne GS, Schwartz K, Bouveret P and Pinset-Härstöm I. Three myosin heavy-chain isozymes appear sequentially in rat muscle development. Nature 292: 805-809, 1981

11. Lyons GE, Ontell M, Cox R, Sassoon D and Buckingham M. The expression of myosin genes in developing skeletal muscle in the mouse embryo. J Cell Biol 111: 1465-1476, 1990

12. Sartorius CA, Lu BD, Acakpo-Satchivi L, Jacobsen RP, Byrnes WC and Leinwand LA. Myosin heavy chains IIa and IId are functionally distinct in the mouse. J Cell Biol 141: 943-953, 1998

13. Hoh JF and Hughes S. Myogenic and neurogenic regulation of myosin gene expression in cat jaw-closing muscles regenerating in fast and slow limb muscle beds. J Muscle Res Cell Motil 9: 59-72, 1988

14. Gojo K, Abe S and Ide Y. Characteristics of myofibres in the masseter muscle of mice during postnatal growth period. Anat Histol Embryol 31: 105-112, 2002

15. Periasamy M, Wieczorek DF and Nadal-Ginard B. Characterization of a developmentally regulated perinatal myosin heavy-chain gene expressed in skeletal muscle. J Biol Chem 259: 13573-13578, 1984

16. Tatsumi R, Anderson JE, Nevoret CJ, Halevy O and Allen RE. HGF/SF is present in normal adult skeletal muscle and is capable of activating satellite cells. Dev Biol 194: 114128, 1998

17. Miller KJ, Thaloor D, Matteson S and Pavlath GK. Hepatocyte growth factor affects satellite cell activation and differentiation in regenerating skeletal muscle. Am J Physiol Cell Physiol 278: 174-181, 2000

18. Rosenthal SM and Cheng ZQ. Opposing early and late effects of insulin-like growth factor I on differentiation and the cell cycle regulatory retinoblastoma protein in skeletal myoblasts. Proc Natl Acad Sci USA 92: 10307-10311, 1995

19. Olson EN. Interplay between proliferation and differentiation within the myogenic lineage. Dev Biol 154: 261-272. 1992

20. Heino J and Massagué J. Cell adhesion to collagen and decreased myogenic gene expression implicated in the control of myogenesis by transforming growth factor beta. J Biol Chem 265: 10181-10184, 1990

21. Rosen GD, Sanes JR, LaChance R, Cunningham JM, Roman $\mathrm{J}$ and Dean DC. Roles for the integrin VLA-4 and its counter receptor VCAM-1 in myogenesis. Cell 69: 1107-1119, 1992

22. Florini JR, Ewton DZ and Magri KA. Hormones, growth factor, and myogenic differentiation. Annu Rev Physiol 53: 201-216, 1991

23. Jennische E, Ekberg S and Matejka GL. Expression of hepatocyte growth factor in growing and regenerating rat skeletal muscle. Am J Physiol 265: C122-C128,1993

24. Schiaffino S, Gorza L, Sartore S, Saggin L, Ausoni S, Vianello M, Gundersen K and Lomo T. Three myosin heavy chain isoforms in type 2 skeletal muscle fibres. J Muscle Res Cell Motil 10: 197-205, 1989

25. Eason JM, Schwartz GA, Pavlath GK and English AW. Sexually dimorphic expression of myosin heavy chains in the adult mouse masseter. J Appl Physiol 89: 251-258, 2000

26. Tuxen A and Kirkeby S. An animal model for human masseter muscle. histochemical characterization of mouse, rat, rabbit, cat, dog, pig, and cow masseter muscle. J Oral Maxillofac Surg 48: 1063-1067, 1990

27. Shida T, Abe S, Sakiyama K, Agematsu H, Mitarashi S, Tamatsu Y and Ide Y. Superficial and deep layer musclefibre properties of the mouse masseter before and after weaning. Arch Oral Biol 50: 65-71, 2005 